\title{
Reemplazo total esofágico mediante ascenso gástrico transmediastinal en tumor del estroma gastrointestinal esofágico: reporte de un caso quirúrgico raro
}

\author{
Total esophageal replacement by transmediastinal transposition of the stomach in a \\ gastrointestinal stromal tumor of the thoracic esophagus: a rare surgical case report
}

\author{
Jesús Morales-Maza ${ }^{1 *}$, Julio Cisneros-Correa ${ }^{1}$, Gabriela Del Ángel-Millán${ }^{1}$, Jorge H. Rodríguez-Quintero', \\ Pablo León-Cabral' y Rubén Cortés-González ${ }^{2}$ \\ ${ }^{1}$ Departamento de Cirugía General; '2Departamento de Cirugía Oncológica. Instituto Nacional de Ciencias Médicas y Nutrición Salvador Zubirán, \\ Ciudad de México, México
}

\section{Resumen}

Antecedentes: Los tumores del estroma gastrointestinal (GIST) son las lesiones malignas mesenquimales más comunes del tracto digestivo. Un sitio raro de localización de estos tumores es el esófago. A la fecha actual no hay un consenso claro basado en evidencia científica con respecto al tipo de cirugía a realizar en pacientes con GIST en esta localización inusual. Caso clínico: Una mujer previamente sana presentó disfagia, pérdida de peso (6 kg) y malestar general. La tomografía computarizada reveló un engrosamiento del esófago y en la ultrasonografía endoscópica se observó una lesión localizada en el esófago dependiente de la muscular propia. Se optó por realizar esofagectomía con reemplazo mediante transposición transmediastinal del estómago. La paciente se recuperó bien de la cirugía y fue dada de alta en condición estable en el séptimo día del posoperatorio. A los 6 meses, se encuentra asintomática. Conclusión: Este caso ilustra la presentación clínica de un GIST esofágico que representa solo el 1\% de todos los sitios reportados. La cirugía se realizó con éxito, con morbilidad mínima, resolución completa de los síntomas y mejoría de la calidad de vida de la paciente. La esofagectomía con reemplazo mediante transposición transmediastinal del estómago (comúnmente llamado ascenso gástrico) debe realizarse cuando el centro tiene experiencia para hacerlo con morbilidad y mortalidad mínimas.

PALABRAS CLAVE: Ascenso gástrico. Cirugía. Esófago. Tumor del estroma gastrointestinal.

\begin{abstract}
Background: Gastrointestinal stromal tumors (GIST) are the most common mesenchymal tumors of the digestive tract. $A$ rare site of localization of these tumors is the esophagus. Evidence-based consensus regarding the type of surgery for patients with esophageal GIST remains unclear. Clinical case: A female without history of diseases experienced dysphagia, weight loss $(6 \mathrm{~kg}$ ) and malaise. Computed tomography revealed thickening of the esophagus. During the endoscopic ultrasonography a localized lesion was observed in the esophagus that depended on the muscularis propria. We opted to treat with an esophagectomy with replacement by transmediastinal transposition of the stomach. Patient recovered well from the surgery and she was discharged home in stable condition in post-operative day seven. At 6 months she has no symptoms. Conclusion: This case illustrates the clinical presentation of an esophageal GIST which represents only $1 \%$
\end{abstract}

\author{
Correspondencia: \\ *Jesús Morales-Maza \\ Vasco de Quiroga, 15 \\ Col. Sección XVI, Del. Tlalpan \\ C.P. 14080 , Ciudad de México, México \\ E-mail: jesus.moralesm1@ hotmail.com
}

Fecha de recepción: 08-08-2018

Fecha de aceptación: 01-07-2019

DOI: 10.24875/CIRU.19000652
Cir Cir. 2019;87:682-687 www.cirugiaycirujanos.com 
of all sites where GISTs have been reported; open surgery was successfully performed with minimal morbidity, complete resolution of symptoms and improvement of the patient's quality of life. Esophagectomy with replacement by transmediastinal transposition of the stomach should be performed when the center has experience to do so with minimal morbidity and mortality.

KEY WORDS: Gastric transposition. Surgery. Esophagus. Gastrointestinal stromal tumor.

\section{Introducción}

Los tumores del estroma gastrointestinal (GIST) son los tumores mesenquimales más comunes del tracto digestivo y provienen de las células de Cajal. En un $80 \%$ de los casos son positivos para marcadores como c-KIT (CD 117), receptor alfa del factor de crecimiento derivado de plaquetas (PDGFRA, platelet-derived growth factor receptor alpha) y CD $34^{1,2}$.

Los sitios más frecuentes donde se encuentran estos tumores son el estómago y el intestino delgado ${ }^{3}$. Se sabe que menos del $1 \%$ de estos tumores se localizan en el esófago, predominantemente en la unión gastroesofágica ${ }^{1,4,5}$, y en general se reportan con mayor frecuencia en hombres de raza blanca ${ }^{6}$.

El tamaño de estos tumores es, en promedio, de 5 a $10 \mathrm{~cm}$ en su mayor eje, pero se han reportado casos de tumores gigantes en el esófago, por lo que, a pesar de ser poco frecuentes, deben considerarse dentro del diagnóstico diferencial de las masas mediastinales ${ }^{7-9}$.

Estos tumores esofágicos suelen tener peor pronóstico en comparación con otros sitios, como el estómago, por ser más grandes. Además, en los análisis mutacionales, el "GIST tipo salvaje» es el más frecuente; es decir, no se encuentran mutaciones en el gen KIT ni en el gen del PDGFRA ${ }^{10}$. La caracterización de estas mutaciones para KIT es muy importante, ya que se sabe que los GIST son resistentes por naturaleza a las quimioterapias convencionales, y desde 2002, cuando se dispuso del inhibidor de la tirosina cinasa imatinib, se observó una gran respuesta al tratamiento inclusive en enfermedad metastásica en los pacientes con estas mutaciones. Es obvio que los GIST esofágicos, al no presentar comúnmente dichas mutaciones, se vuelven más resistentes a estas terapias y por consiguiente presentan peor pronóstico ${ }^{11}$.

La resección quirúrgica es el tratamiento de elección en todos los casos, ya que mejora la supervivencia de los pacientes a largo plazo ${ }^{12}$.

Presentamos un caso de GIST esofágico en el que se realizó esofagectomía con reemplazo esofágico mediante ascenso gástrico.

\section{Caso clínico}

Se trata de una mujer de 29 años sin antecedentes de enfermedades, con un cuadro de 1 año de evolución de disfagia esofágica para sólidos y líquidos, dolor retroesternal de característica opresiva, constante y de intensidad 4/10 que empeoraba con los alimentos. Además, presentaba regurgitación de los alimentos 10-15 minutos después de la ingestión, pérdida de peso $(6 \mathrm{~kg})$ en los últimos 6 meses y malestar general. A la exploración física no se observaban alteraciones.

Se complementó el abordaje diagnóstico con tomografía computarizada que reveló engrosamiento de la porción torácica del esófago. No se encontraron otras anormalidades en ese estudio. Se realizó endoscopia gastrointestinal con ultrasonografía endoscópica, que mostraron una lesión esofágica submucosa dependiente de la cuarta endocapa de la pared esofágica (muscular propia) sin evidencia de adenopatías sospechosas ni extensión extraesofágica (Fig. 1). Se sospechó el diagnóstico probable de tumor del estroma gastrointestinal esofágico.

Se realizó esofagectomía con reemplazo gástrico transmediastinal mediante doble abordaje (abdominal y cervical). El abordaje abdominal inicial se realizó con una disección del epiplón mayor, conservando las arterias gastroepiploicas y gástrica derecha con ligadura de la arteria gástrica izquierda y los vasos cortos. La disección esofágica se realizó a través del hiato esofágico, con liberación de las fijaciones del mediastino. Se realizó vagotomía troncal bilateral.

La fase cervical se realizó con una incisión en forma de $\mathrm{J}$ en el borde anterior del músculo esternocleidomastoideo izquierdo. Se identificaron las estructuras vasculares cervicales importantes (vena yugular interna y arteria carótida común), así como el nervio laríngeo recurrente izquierdo, que se respetaron durante todo el procedimiento. Se realizó la disección del espacio retroesofágico utilizando una engrapadora lineal cortante de $45 \mathrm{~mm}$ (Fig. 2).

Una vez que el esófago se liberó completamente hacia la porción abdominal, se realizaron la esofagectomía 

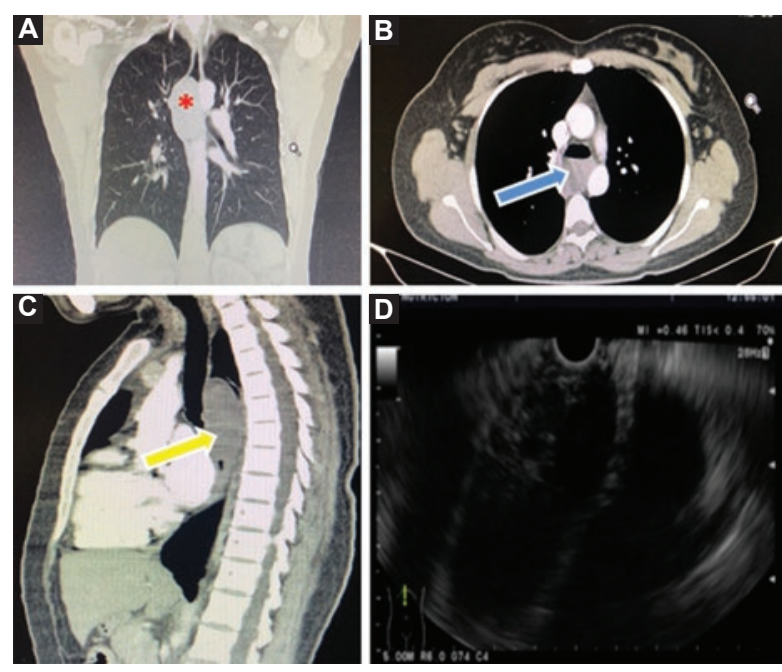

Figura 1. A: corte coronal de la tomografía computarizada (TC) de tórax que muestra la lesión dependiente del tercio superior del esófago con un ligero desplazamiento traqueal hacia la derecha. La lesión está marcada con un asterisco rojo; B: corte axial de la TC que muestra la lesión esofágica señalada con una flecha azul; C: corte sagital que muestra la lesión esofágica señalada con una flecha amarilla; D: evidencia de la lesión esofágica mediante ultrasonografía endoscópica.

y la tubulización del estómago con tres cartuchos azules de engrapadora lineal cortante de $75 \mathrm{~mm}$. Se reforzó la línea de grapas con sutura continua usando Prolene ${ }^{\circledR}$ 3-0 (Fig. 3).

El ascenso gástrico se llevó a cabo pasando la parte tubulizada del estómago hacia la región cervical de forma retroesternal y finalmente se realizó la anastomosis esofagogástrica manual término-terminal. Como se realizó una vagotomía troncal bilateral fue necesario hacer una piloroplastia tipo Heineke-Mikulicz, la cual consiste en una incisión longitudinal de aproximadamente $4-5 \mathrm{~cm}$ del píloro y cierre mediante 6-8 puntos separados de manera transversal en dos capas, con la finalidad de ampliar la luz pilórica, prevenir un trastorno de la motilidad gastroduodenal y favorecer el vaciado gástrico.

Debido a que es una anastomosis "alta», con un mayor riesgo de fuga en comparación con otros sitios de anastomosis, se consideró prudente realizar una yeyunostomía de alimentación para así evitar la vía oral y proteger la anastomosis realizada al menos durante las primeras 2 semanas. Se colocaron dos drenajes tipo Blake \#19 en el hiato esofágico y otro drenaje \#15 en la región cervical. Aunque existe debate sobre la colocación de drenajes, se decidió colocarlos porque tienen la característica de ser cerrados activos y al tratarse de una cirugía extensa podrían prevenir la acumulación de líquido en el espacio muerto. Por otro lado, la anastomosis esofágica tiene con mayor frecuencia un «alto riesgo» de fugas en comparación con cualquier otro sitio de anastomosis gastrointestinal, así que el drenaje podría alertarnos sobre una posible fuga y al mismo tiempo drenar el líquido de fuga sin necesidad de más tratamiento.

La duración de la cirugía fue de 300 minutos y el sangrado quirúrgico fue de $50 \mathrm{ml}$. Durante toda la intervención la paciente se encontró hemodinámicamente estable y con buena perfusión tisular, por lo que al término de esta pasó al área de recuperación sin ameritar su ingreso a un área de cuidados intensivos. Desde el día 1 del posoperatorio se insistió en la realización de inspirometría, oxigenación mediante puntas nasales y deambulación asistida por sus familiares. Se inició profilaxis antitrombótica con heparina no fraccionada a dosis de $5000 \mathrm{UI}$ cada 12 horas a partir de las primeras 12 horas del término de la cirugía. La estancia hospitalaria fue de 7 días, sin ninguna eventualidad. La yeyunostomía se retiró a las 6 semanas de la cirugía, en el consultorio.

El informe de patología definitivo fue un tumor del estroma gastrointestinal de $6 \times 5 \times 2 \mathrm{~cm}$ en el tercio superior del esófago torácico. El índice de mitosis fue de 0 en 10 campos de alto poder, sin necrosis, con márgenes quirúrgicos negativos. La inmunohistoquímica reportó CD $117+$, actina +, desmina +, DOG 1 - y calponina -, lo que confirmó el diagnóstico de GIST.

Actualmente la paciente se encuentra en el sexto mes del seguimiento posoperatorio y se realizan revisiones periódicas cada 2 meses. Manifiesta buena tolerancia de la vía oral y sin episodios de regurgitación, reflujo ni dolor torácico. Ha aumentado $5 \mathrm{~kg}$ de peso desde la cirugía. Continúa su seguimiento por los servicios de gastroenterología, nutriología, cirugía oncológica y oncología médica. Este último servicio indicó tratamiento adyuvante con imatinib, $400 \mathrm{mg}$ al día durante 3 años, debido al riesgo de recurrencia específico de la paciente $(35 \% \text { a } 5 \text { años })^{13}$.

\section{Discusión}

El esófago es uno de los sitios más raros donde se reportan tumores del estroma gastrointestinal, con una frecuencia menor del 1\% en relación con las demás localizaciones.

Al ser una afección rara, en la literatura solo pueden encontrarse reportes de casos, y por esta razón es difícil contar con una descripción exacta de qué hacer con casos especiales como el que aquí se presenta ${ }^{14}$. 

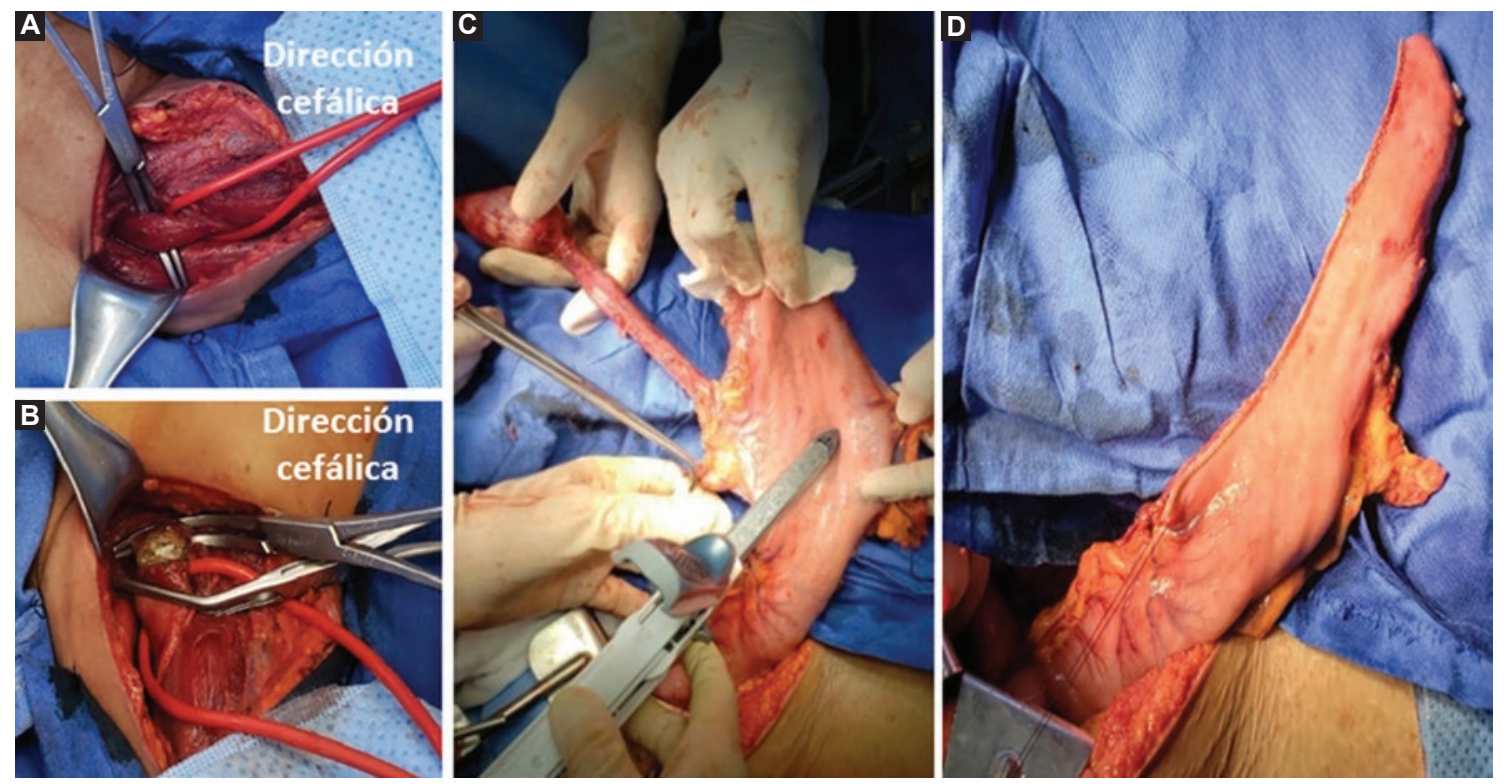

Figura 2. A: fase cervical, disección del esófago; B: sección del esófago cervical; C: fase abdominal, «tubulización» del estómago utilizando engrapadora lineal cortante; D: estómago en forma tubular listo para el ascenso gástrico transmediastinal.
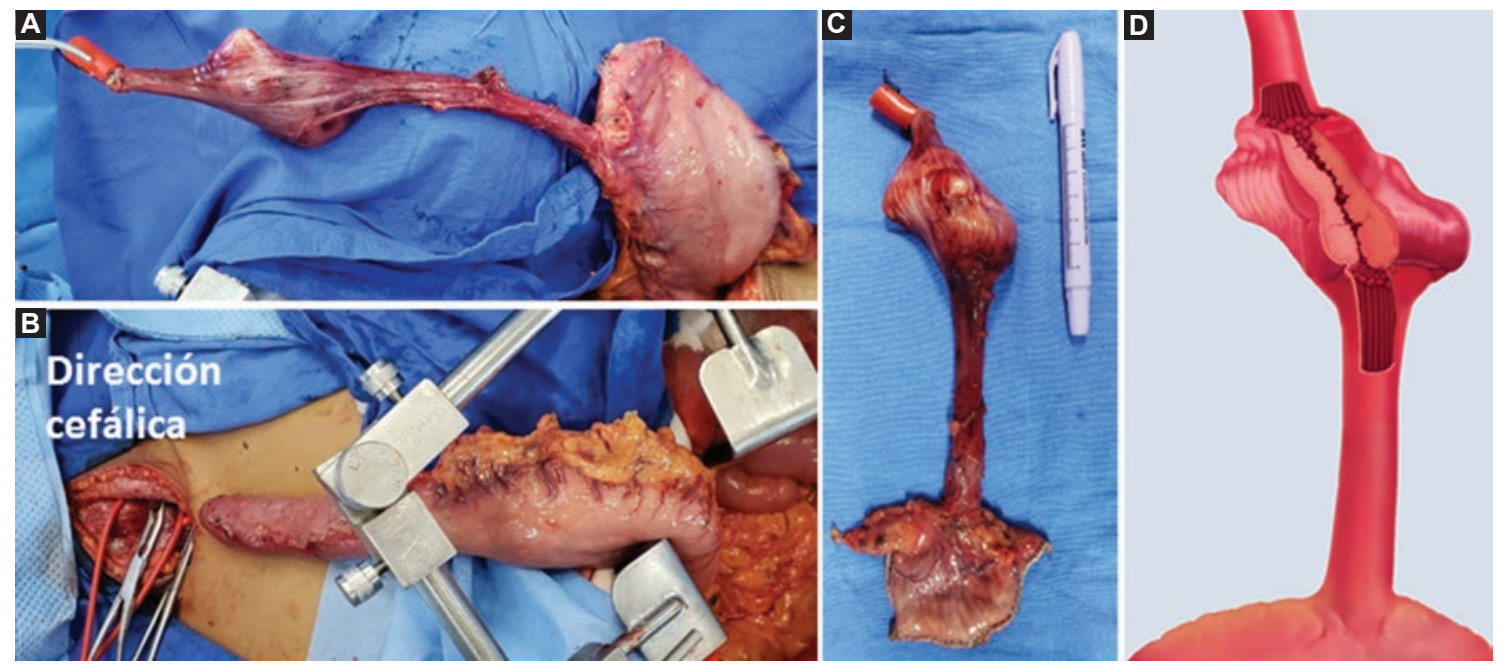

Figura 3. A: esófago liberado completamente en la cavidad abdominal con evidencia de la masa esofágica; B: estómago con porción tubulizada hacia la región cervical; C: pieza quirúrgica de la esofagectomía que incluye un segmento de la unión esofagogástrica; D: representación gráfica que muestra el GIST esofágico.

Aunque se han descrito diferentes técnicas quirúrgicas de esofagectomía, que incluyen resección mediante toracotomía, cirugía toracoscópica video-asistida (VATS), laparoscopia y abordajes híbridos que combinan la endoscopia con la toracoscopia, en algunos casos, como el reportado, se prefiere realizar un doble abordaje, abdominal y cervical, con reemplazo gástrico, ya que no se recomienda la simple enucleación en tumores mayores de $2 \mathrm{~cm}$ por el riesgo de rotura del tumor, que confiere al paciente un mayor riesgo de recurrencia ${ }^{15-18}$.

Esta cirugía con disección esofágica sin toracotomía fue descrita en 1936 por Turner ${ }^{19}$ para el tratamiento del carcinoma en el esófago torácico, y en 1971 fue perfeccionada por Akiyama, et al. ${ }^{20}$. Debe destacarse que en un inicio no se recomendaba para el cáncer esofágico por dos motivos principales: por realizar disección roma del esófago con los dedos, lo cual aparentaba incrementar el riesgo de sangrado, y por tener la desventaja de no poder realizarse una disección ganglionar formal ${ }^{21}$. Sin embargo, muchos autores han logrado reproducir dicha técnica con muy buenos resultados y sin evidenciar altos porcentajes de complicaciones. Orringer, et al. ${ }^{22}$ reportaron su serie de más de 1000 casos operados con fuga de anastomosis en el $13 \%$, atelectasias o neumonía en el $2 \%$, 
hemorragia intratorácica, lesión del nervio laríngeo recurrente, quilotórax o laceración traqueal en menos del $1 \%$, y muy buenos resultados funcionales en el $70 \%$ de los casos. Estos autores reportan una estancia hospitalaria de 7 días, igual que en nuestro caso. Estos resultados convierten esta técnica quirúrgica en un procedimiento más seguro y con menos complicaciones que los procedimientos en los que se realiza abordaje mediante toracotomía ${ }^{22}$.

Esta técnica quirúrgica debe realizarse cuando el centro tiene experiencia para hacerlo con una morbilidad y una mortalidad mínimas. Consideramos que nuestro centro tiene buena experiencia en la realización de estos procedimientos, ya que es un centro de tercer nivel de referencia de enfermedades esofágicas neoplásicas y no neoplásicas. Se tienen cuantificados al menos 70 casos de esofagectomías y específicamente 59 con reconstrucción mediante ascenso gástrico transmediastinal, siendo el resto de las reconstrucciones mediante transposición colóni$\mathrm{Ca}^{23}$. Cuando contemplamos la morbimortalidad total de las esofagectomías mediante ambos tipos de reconstrucción, es alta: más del $85 \%$ con evidencia de derrame pleural en el $37 \%$, neumonía en el $7 \%$, atelectasia y mediastinitis en el $5 \%$, fuga de anastomosis cervical en el $42 \%$ (todos los casos fueron manejados de manera conservadora), estenosis de la anastomosis en el $32 \%$ (todos los casos fueron manejados con tratamiento endoscópico con dilatación), infección de sitio quirúrgico superficial en el $57 \%$ y lesión del nervio laríngeo recurrente en el $4 \%$. La mortalidad a los 30 días del posoperatorio reportada en nuestro centro es del $4.28 \%$. Debe señalarse que hay que ser cautelosos al analizar estos resultados, ya que no contamos con los resultados específicamente de la reconstrucción mediante ascenso gástrico y, al realizar el análisis completo incluyendo la reconstrucción mediante transposición colónica, los porcentajes de complicaciones se sobreestiman.

\section{Conclusión}

Este caso ilustra la presentación clínica de un GIST esofágico, que es un sitio de localización inusual. La esofagectomía con ascenso gástrico se realizó con éxito, logrando la resolución completa de los síntomas y una mejoría de la calidad de vida de la paciente.

La esofagectomía con reemplazo total esofágico mediante ascenso gástrico transmediastinal en el tratamiento del GIST esofágico es una buena opción quirúrgica con resultados satisfactorios. Esta técnica debe realizarse cuando el centro tiene experiencia para hacerlo con una morbilidad y una mortalidad mínimas.

\section{Agradecimientos}

Agradecimiento especial al Licenciado en Diseño Gráfico Diego Díaz Campos por haber realizado el gráfico del GIST esofágico.

\section{Financiamiento}

Los autores declaran no recibir ningún financiamiento.

\section{Conflicto de intereses}

Los autores declaran no tener ningún conflicto de intereses.

\section{Responsabilidades éticas}

Protección de personas y animales. Los autores declaran que los procedimientos seguidos se conformaron a las normas éticas del comité de experimentación humana responsable y de acuerdo con la Asociación Médica Mundial y la Declaración de Helsinki.

Confidencialidad de los datos. Los autores declaran que han seguido los protocolos de su centro de trabajo sobre la publicación de datos de pacientes.

Derecho a la privacidad y consentimiento informado. Los autores han obtenido el consentimiento informado de los pacientes y/o sujetos referidos en el artículo. Este documento obra en poder del autor de correspondencia.

\section{Bibliografía}

1. Hihara J, Mukaida H, Hirabayashi N. Gastrointestinal stromal tumor of the esophagus: current issues of diagnosis, surgery and drug therapy. Transl Gastroenterol Hepatol. 2018;3:6.

2. von Mehren M, Randall RL, Benjamin RS, Boles S, Bui MM, Casper ES, et al. Gastrointestinal stromal tumors, version 2.2014. J Natl Compr Canc Netw. 2014;12:853-62.

3. Al-Jiffry BO, Allam HM, Hatem M. Single-center experience of surgically resected gastrointestinal stromal tumors: a report of six cases, including a rare case involving the lower esophagus. Oncol Lett. 2015;9:745-8.

4. Søreide K, Sandvik OM, Søreide JA, Giljaca V, Jureckova A, Bulusu VR. Global epidemiology of gastrointestinal stromal tumours (GIST): a systematic review of population-based cohort studies. Cancer Epidemiol. 2016;40:39-46.

5. Mu ZM, Xie YC, Peng XX, Zhang H, Hui G, Wu H, et al. Long-term survival after enucleation of a giant esophageal gastrointestinal stromal tumor. World J Gastroenterol. 2014;20:13632-6.

6. Kukar M, Kapil A, Papenfuss W, Groman A, Grobmyer SR, Hochwald SN Gastrointestinal stromal tumors (GISTs) at uncommon locations: a large population based analysis. J Surg Oncol. 2015;111:696-701.

7. Duffaud F, Meeus P, Bertucci F, Delhorme JB, Stoeckle E, Isambert N, et al. Patterns of care and clinical outcomes in primary oesophageal gastrointestinal stromal tumours (GIST): a retrospective study of the French Sarcoma Group (FSG). Eur J Surg Oncol. 2017;43:1110-6. 
8. Nakano A, Akutsu Y, Shuto K, Uesato M, Kono T, Hoshino I, et al. Giant esophageal gastrointestinal stromal tumor: report of a case. Surg Today. 2015;45:247-52.

9. Kafeel M, Cheedella NK, Wang JC. Esophageal gastrointestinal stromal tumors presenting as mediastinal mass. Case Rep Oncol. 2013;6:579-84.

10. Lott S, Schmieder M, Mayer B, Henne-Bruns D, Knippschild U, Agaimy A, et al. Gastrointestinal stromal tumors of the esophagus: evaluation of a pooled case series regarding clinicopathological features and clinical outcome. Am J Cancer Res. 2014;5:333-43.

11. Lott S, Schmieder M, Mayer B, Henne-Bruns D, Knippschild U, Agaimy A, et al. Gastrointestinal stromal tumors of the esophagus: evaluation of a pooled case series regarding clinicopathological features and clinical outcome. Am J Cancer Res. 2014;5:333-43.

12. Zhang FB, Shi HC, Shu YS, Shi WP, Lu SC, Zhang XY, et al. Diagnosis and surgical treatment of esophageal gastrointestinal stromal tumors. World J Gastroenterol. 2015;21:5630-4.

13. Racz JM, Brar SS, Cleghorn MC, Jimenez MC, Azin A, Atenafu EG, et al. The accuracy of three predictive models in the evaluation of recurrence rates for gastrointestinal stromal tumors. J Surg Oncol. 2015;111:371-6.

14. Wada $Y$, Kadokura M, Kamio $Y$, Kitami A, Nakajima $H$, Inoue $H$, et al. Esophageal gastrointestinal stromal tumor surrounding the middle esophagus with dysphagia for 8 years; report of a case. Kyobu Geka. 2004;57:1250-3.
15. Constantinoiu S, Gheorghe M, Popa L, Ciocea C, losif C, Tiutiuca R, et al. Giant esophageal GIST: diagnostic and therapeutic challenge case report. Chirurgia (Bucur). 2015;110:300-7.

16. Nawara C, Augschöll C, Hutter J, Ofner D, Primavesi F. Oesophageal GIST at the left tracheobronchial angle: resection with right-sided VATS. Zentralbl Chir. 2013;138:499-501.

17. Daiko H, Fujita T, Ohgara T, Yamazaki N, Fujii S, Ohno Y, et al. Minimally invasive hybrid surgery combined with endoscopic and thoracoscopic approaches for submucosal tumor originating from thoracic esophagus. World J Surg Oncol. 2015;13:40.

18. Blum MG, Bilimoria KY, Wayne JD, de Hoyos AL, Talamonti MS, Adley B. Surgical considerations for the management and resection of esophageal gastrointestinal stromal tumors. Ann Thorac Surg. 2007;84:1717-23.

19. Turner GG. Carcinoma of the oesophagus. The question of its treatment by surgery. Lancet. 1936;1:130-4.

20. Akiyama H, Sato $Y$, Takashashi F. Immediate pharyngogastrostomy following total esophagectomy by blunt dissection. Jpn J Surg. 1971;1:225-31.

21. Akiyama $H$, Hiyama $M$, Miyazono $H$. Total esophageal reconstruction after extraction of the esophagus. Ann Surg. 1975;182:547-52.

22. Orringer MB, Marshall B, lannettoni MD. Transhiatal esophagectomy: clinical experience and refinements. Ann Surg. 1999;230:392-400.

23. Mejía-Rivera S, Pérez-Marroquín SA, Cortés-González R, Medina-Franco H. Contrast-enhanced swallow study sensitivity for anastomotic leak detection in post-esophagectomy patients. Rev Gastroenterol Mex. 2018;83:400-4. 